\title{
Wireless data transfer through biological tissues using near-infrared light: testing skull and skin phantoms
}

Ahmed, Iqrar, Bykov, Alexander, Popov, Alexey, Meglinski, Igor, Katz, Marcos

Igrar Ahmed, Alexander Bykov, Alexey Popov, Igor Meglinski, Marcos Katz, "Wireless data transfer through biological tissues using near-infrared light: testing skull and skin phantoms," Proc. SPIE 11226, Neural Imaging and Sensing 2020, 1122615 (21 February 2020); doi: 10.1117/12.2545221

SPIE. Event: SPIE BiOS, 2020, San Francisco, California, United States 


\title{
Wireless Data Transfer Through Biological Tissues Using Near-infrared Light: Testing Skull and Skin Phantoms
}

\author{
Iqrar Ahmed ${ }^{\mathrm{a}}$, Alexander Bykov ${ }^{\mathrm{b}}$, Alexey Popov ${ }^{\mathrm{b}}$, Igor Meglinski ${ }^{\mathrm{b}}$, and Marcos Katz \\ ${ }^{a}$ Center for Wireless Communications, University of Oulu, Oulu, Finland \\ ${ }^{\mathrm{b}}$ Opto-Electronics and Measurement Techniques, University of Oulu, Oulu, Finland
}

\begin{abstract}
Light in the range of 700-1100 $\mathrm{nm}$ has many proven diagnostics and therapeutic medical applications. In this study, we demonstrate another potential application of light. We implemented a testbed to transmit data wirelessly through a human skull and biological phantoms mimicking skin tissues properties using near-infrared (NIR) light. Currently, radio frequency (RF) is widely used to transmit data wirelessly through biological tissues, example includes implants communications. However, RF communications contain interference and security challenges. In this study, we present a novel use of NIR light for wireless data transfer, for which a phantom is exposed to an externally modulated light source and the transmitted light through phantom is captured with a receiver. Initially, a high-resolution picture is successfully transmitted through skin phantom of $2 \mathrm{~cm}$ in depth. In the second phase, we successfully communicated through a human skull. Due to hardware limitations, we are able to achieve data rates in tens of kilobits per second. The minimum power applied to the phantoms measured during the data transmission from $810 \mathrm{~nm}$ light-emitting-diode (LED) is $0.098 \mathrm{~W} / \mathrm{cm} 2$. The measured optical power values are within the safe limit set by ANSI.Z136.1-2007 standard on laser safety, which is $2 \mathrm{~W} / \mathrm{cm}^{2}$ for 1 sec exposure of $830 \mathrm{~nm}$ wavelength. Initial results are encouraging and there are many possible applications for a system using light to transmit data wirelessly through biological tissues, including communication with brain implants and performing brain therapies.
\end{abstract}

Keywords: MICS, RF, Bio tissues, OWC, Near-infrared

\section{INTRODUCTION}

Since its development, the Radio frequency $(\mathrm{RF})$ is predominant technology being used to transmit data wirelessly. In practise, a conductive coupling is first utilized to gather information from living organism. A typical example is registering the electrocardiograms (ECG) using $\mathrm{Ag} / \mathrm{AgCl}$ electrodes. When transmitting information from outside to an implant placed inside the living organism, an inductive communication is the conventional way. But the conventional inductive type communication has limitations including very low distance between the outside device and the implant. Secondly, the additional electromagnetic interference from the surrounding devices. Unlike inductive, the RF is utilized in wireless medical device communication 1. Medical Implant Communication Service (MICS) and Medical Device Radio communications Service(MedRadio) use the RF to communicate with implants either for drug delivery or for diagnostic purposes. Though minimum SAR with biological effect (MSBE) has been studied and devices utilizing RF must meet the lower minimum requirements for safety 2,3 , the challenges with RF such as security and safety persist. Studies shows that implants are vulnerable to security threats 4 . In this study, we propose and demonstrate a novel technique of wirelessly transmitting data through biological tissues, using near-infrared (NIR) light. For which we illuminated phantoms with Thorlabs $810 \mathrm{~nm}$ NIR LED and successfully transmitted couple of centimeters through biological phantom mimicking human skin and skull. We present our work in detail.

Further author information: (Send correspondence to)

Iqrar Ahmed

E-mail: iqrar.ahmed@oulu.fi, Telephone: +358 407508708

Neural Imaging and Sensing 2020, edited by Qingming Luo, Jun Ding, Ling Fu, Proc. of SPIE

Vol. 11226, 1122615 - (c) 2020 SPIE · CCC code: 1605-7422/20/\$21 - doi: 10.1117/12.2545221 


\section{SYSTEM OVERVIEW}

Light has many applications in medical practices ranging from diagnostics to drug delivery, etc. Light has potential benefits over RF including security i.e., light signal can't be hack remotely and safety i.e., no radiation exposure as we know that ionizing radiation could alter the molecular biology. Since NIR and laser lights are mostly use for medical applications, longer or stronger exposures of these therapeutic light sources could result in adverse outcomes. ICNIRP provides the guidelines needed for human safety 5 . We implemented our testbed utilizing off-the-shelf electronics.A block diagram of our testbed is shown in Figure 1.

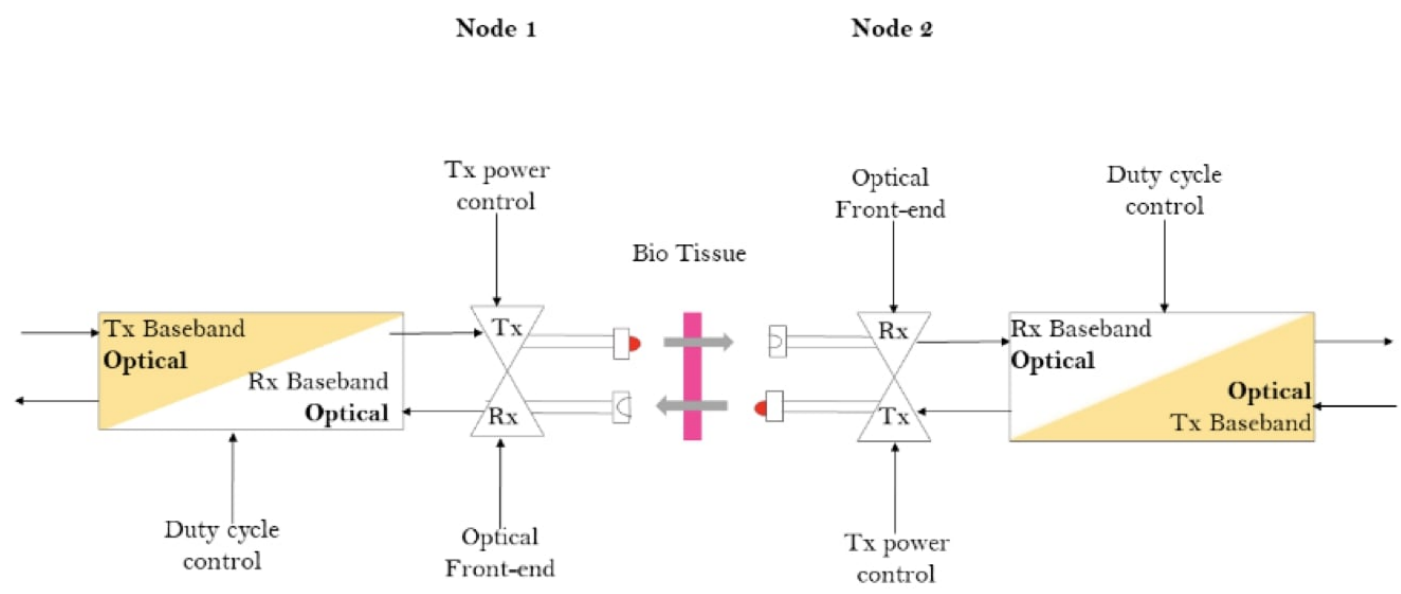

Figure 1. Block diagram of implemented testbed.

Transmitter and receiver nodes are constructed using GNU radio software on Linux PCs, which is free to use software. Different parameters such as modulation scheme, transmit and receive frequencies etc., are selected accordingly in GNU radio software and data from transmitter PC is then sent to the connecting Universal Software Radio Peripheral(USRP) device to generate a baseband signal. The baseband modulated signal from USRP is fed to Bias-T to add biasing between 0 to $3.6 \mathrm{~V}$, because the LED we are using operates in this range. The DC biased modulated baseband signal is then applied to a LED driver which generates the equivalent current. The modulation coefficient is predefined that converts the input voltage to LED driver into equivalent current. The specification sheet tells that driver produces $400 \mathrm{~mA}$ for $1 \mathrm{~V}$ applied through externally modulated signal form Bias-T. The LED operates in response to the applied modulated signal, the light transmitted through biological tissue is captured on the opposite with a photo diode receiver. The receiver is connected to USRP and GNU radio software, which demodulate the received signal and present on screen, in our case an image is reconstructed transitionally.

\subsection{Transmitter and Receiver Electronics}

We have used Thorlabs electronics to construct transmitter and receiver frontends. The transmitter contains the NIR LED, LED driver and focusing lens. We have used $810 \mathrm{~nm}$ mounted IR LED 6, LED driver 7 is capable of handling externally modulated signal. The beam confinement is performing by anti-reflective (AR) lens 8 . The detector 9, is a photodiode receiver operate within the range of $400 \mathrm{~nm}$ to $1000 \mathrm{~nm}$. An overview of the implemented testbed is shown in Figure 2. A receiver is placed underneath the human skull, the information is transmitted through skull. A detector connected to power meter is used to estimate the amount of light. We estimated the power transmitted from the LED by placing detector at the LED and power received by placing detector at the skull. Phantoms of variable depth are placed on the skull to mimic the human head. 


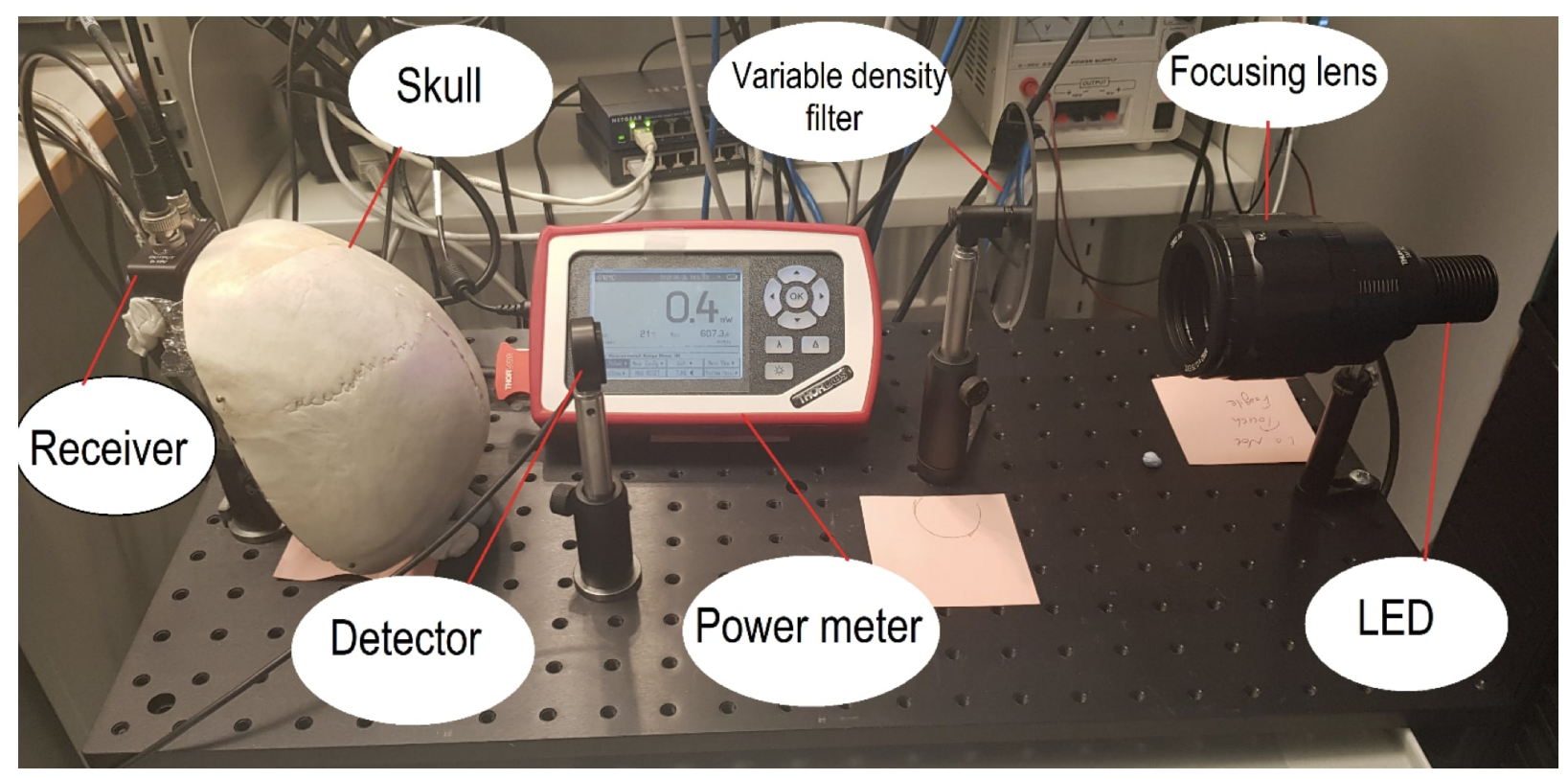

Figure 2. Testbed overview.

\section{TESTING}

Extensive measurements are needed when estimating the endogenous or haemodynamics properties of bio tissues. Since the pathology and endogenous properties of sample bio tissues degrades over time the need of bio tissues mimicking phantoms are required for testing in laboratories. The phantoms we used for experimentation were fabricated in our labs by our opto-electronics team 10,11. The skin phantoms we used We added multiple layers of phantoms to mimic the variable skin depth. We also used the combinations of skin phantoms and skull to mimic the human head.

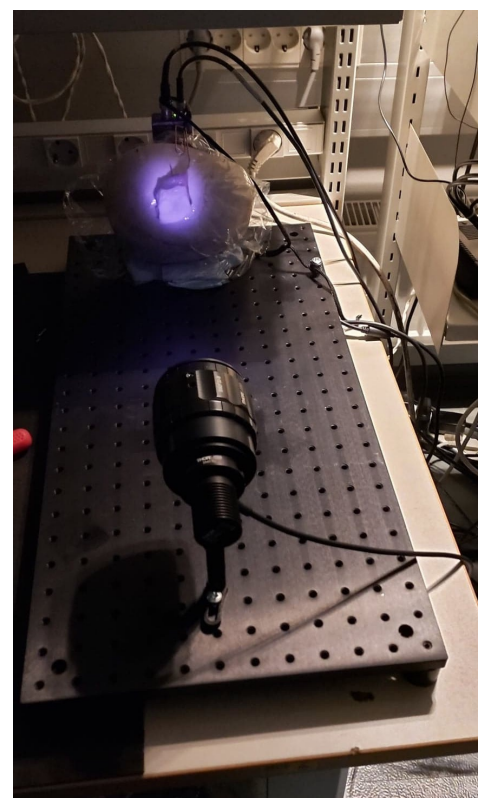

Figure 3. Laboratory setting for OCBT. 
We transmitted an image through the skull and skin phantoms placed on the skull. Another testing is performed with pork meat, we used chunks of pork meat and fat of variable sizes. The pork meat is first heated to $37^{\circ} \mathrm{C}$, the variable thicknesses are then tested by placing those onto the skull. We varied the distance between the light source and the receiver to check the drop in transmission along with distance. Figure 3 shows the settings of source and receiver, the light is focused onto the chunk of pork meat placed on the skull placed laterally.

\section{LIMITATIONS}

We present a novel optical communication through bio tissue (OCBT) technique using the NIR light. There are many possible use cases for OCBT, it can be used to communicate with pacemaker, therapeutic and drug delivery implants. The potential benefits of OCBT over RF communications include Security and Safety. OCBT is potentially secure as it requires line-of-site connection and can't be hack remotely and has no radiation exposure issues compare to radio frequency $(\mathrm{RF})$ communications. We successfully transmitted data through skull and skin phantoms but certain limitations are observed during experimentation. These limitations are mainly due to hardware, since we are using off-the-shelf electronics from Thorlabs, we faced limitation in achieved data rate. So far, we have achieved tens of Kbits/s, this low data rate is sufficient for transmitting small amount of medical data i.e., profiles, files, etc. In our case, we successfully transmitted a high-resolution image.

\section{RESULTS AND CONCLUSIONS}

We measured the optical power given to the phantom and received optical power as shown in Table 1. According to ANSI.Z136.1-2007 standard on laser safety,an exposure of $1 \mathrm{sec}$ of $830 \mathrm{~nm}$ wavelength that can produce 2 $\mathrm{W} / \mathrm{cm}^{2}$ power is considered safe. In this study, we measured the maximum power given to the phantoms is 0.10 $W / \mathrm{cm}^{2}$, which is well within the safe limit.

Table 1. The amount of optical power delivered to phantom versus the received power.

\begin{tabular}{|c|c|c|}
\hline Depth of Phantom $(\mathrm{mm})$ & Optical Power to Phantom $(\mathrm{mW} / \mathrm{cm} 2)$ & Received Optical Power $(\mathrm{mW} / \mathrm{cm} 2)$ \\
\hline 4 & 98.2 & 0.0067 \\
\hline 6 & 102.7 .94 & 0.0071 \\
\hline 10 & 106.3 & 0.0078 \\
\hline
\end{tabular}

The demonstrated OCBT is simple and inexpensive technology that offers potential benefits of security and safety over the RF. Several challenges are to be overcome in OCBT including, embedding the proposed technology into system-on-chip (SoC). The potential benefits of security and user safety in OCBT make the demonstrated system a potential future medical application.Further research is required to achieve higher data rates and testing different tissue types.

\section{REFERENCES}

[1] Islam, M. N. and Yuce, M. R., "Review of medical implant communication system (mics) band and network," Ict Express 2, 188-194 (2016).

[2] International Commission on Non-Ionizing Radiation Protection, ., "Limiting exposure to time-varying electric and magnetic fields (1 hz to $100 \mathrm{khz}$ ). health physics, 99(6), pp.818-836 (2010)."

[3] Lin, J. C., "Safety standards for human exposure to radio frequency radiation and their biological rationale," IEEE Micro. Mag. 4, 22-26 (2003).

[4] Pycroft, L. and Aziz, T. Z., "Security of implantable medical devices with wireless connections: The dangers of cyber-attacks," Ict Express , 403-406 (2018).

[5] International Commission on Non-Ionizing Radiation Protection, ., "ICNIRP guidelines on limits of exposure to incoherent visible and infrared radiation. Health Physics, 105(1), pp.74-96.."

[6] https://www.thorlabs.com/thorproduct.cfm?partnumber=M810L3. 
[7] https://www.thorlabs.com/newgrouppage9. cfm?objectgroup_id=9117\&pn=DC2200\#9171.

[8] https://www.thorlabs.com/thorproduct.cfm?partnumber=SM2F32-B.

[9] https://www.thorlabs. com/thorproduct.cfm?partnumber=PDA015A.

[10] Wróbel, M., Popov, A., Bykov, A., Kinnunen, M., Jedrzejewska-Szczerska, M., and Tuchin, V., "Multilayered tissue head phantoms for noninvasive optical diagnostics," J. Innov. Opt. Healt. Scien. 8, 1541005 (2015).

[11] Wróbel, M., Popov, A., Bykov, A., Kinnunen, M., Jedrzejewska-Szczerska, M., and Tuchin, V., "Measurements of fundamental properties of homogeneous tissue phantoms," J. biomed. opt. 20, 045004 (2015). 